\title{
A time-dependent genome-wide SNP-SNP interaction analysis of chicken body weight
}

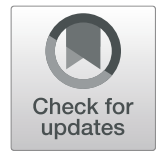

Fang-Ge Li ${ }^{1,2,3}$ and Hui Li, ${ }^{2,3,4^{*}}$

\begin{abstract}
Background: The important property of the quantitative traits of model organisms is time-dependent. However, the methodology for investigating the genetic interaction network over time is still lacking. Our study aims to provide insights into the mechanistic basis of epistatic interactions affecting the phenotypes of model organisms.

Results: We performed an exhaustive genome-wide search for significant SNP-SNP interactions associated with male birds' body weight (BW) $(n=475)$ at multiple time points (day of hatch (BW0) and 1, 3, 5, and 7 weeks (BW1, BW3, BW5, and BW7)). Statistical analysis detected 67, four, and two significant SNP pairs associated with BW0, BW1, and BW3, respectively, with a significance threshold at $8.67 \times 10^{-12}$ (Bonferroni-adjusted: 1\%). Meanwhile, no significant SNP pairs associated with BW5 and BW7 were found. The SNP-SNP interaction networks of BW0, BW1, and BW3 were built and annotated.
\end{abstract}

Conclusions: With strong annotated information and a strict significant threshold, SNP-SNP interactions underpinned the gene-gene interactions that might occur between chromosomes or within the same chromosome. Comparing and combing the networks, the results indicated that the genetic network for chicken body weight was dynamic and time-dependent.

\section{Background}

Epistatic interactions (non-linear interactions between segregating loci) are gaining attention in contemporary biology, yet their role in the genetic architecture of quantitative traits is still obscure and controversial. Studies on fruit fly (Drosophila melanogaster), yeast (Saccharomyces cerevisiae), mouse (Mus musculus), thale cress (Arabidopsis thaliana), maize (Zea mays), and human (Homo sapiens) demonstrate that epistasis is pervasive and is an important factor in determining the variation of quantitative phenotypes [1-4]. On the other hand, in the past 15 years, thousands of genome-wide association studies have reported numerous single SNP loci that exhibit significant additive effects; however, especially for quantitative traits and complex diseases, the results were challenged for missing heritability. A typical example is human

\footnotetext{
* Correspondence: lihui@neau.edu.cn

${ }^{2}$ Key Laboratory of Chicken Genetics and Breeding, Ministry of Agriculture and Rural Affairs, Harbin 150030, People's Republic of China

${ }^{3}$ Key Laboratory of Animal Genetics, Breeding and Reproduction, Education Department of Heilongjiang Province, Harbin 150030, People's Republic of China

Full list of author information is available at the end of the article
}

height, which is a classic quantitative trait with an estimated heritability of about $80 \%$, and has been associated with more than 700 SNP loci, which, however, explain only about $20 \%$ of heritability $[5,6]$. Geneticists have postulated that identifying epistatic interactions between SNP loci would be a reasonable way to explain heritable variance [7]. Some studies have clarified this idea, including the study by Zuk et al. that demonstrated that a large part of the missing heritability of Crohn's disease could be due to genetic interactions [8].

Carlborg et al. revealed that an apparently major locus for chicken growth belonged to a genetic network of four interacting loci, which indicates that epistatic interactions between genes (or quantitative trait loci) were important for quantitative traits in chicken [9]. Furthermore, our previous studies also detected epistatic interactions and demonstrated that they could affect the variation in chicken phenotypes [10-12].

In the current study, we focused on the body weight of chickens whose phenotypic data could be analyzed as a series of data points (i.e. time series). The chickens' 
lifespan was divided into four equal periods of 2 weeks. The body weight at five time points was selected as a phenotypic value. Significant SNP-SNP interactions associated with the body weights at different weeks (BW0, BW1, BW3, BW5, and BW7) were detected with an exhaustive genome-wide test. Next, the SNP-SNP interaction networks were built and annotated. Our results provide further insight into the genetic network that controls body weight in chickens.

\section{Results}

\section{SNP genotyping and phenotypic values}

After quality control, the following was included in this study: a total of 48,152 SNPs on 28 autosomes, the Z chromosome, linkage groups, and 672 SNPs not assigned to any chromosomes in chickens (Table 1). Finally, 48,034 SNPs with chromosome position information were filtered for the interaction analysis.

Phenotypic descriptive statistics for body weight are listed in Table 2. The body weights exhibited no significant differences between the lean and fat lines, so we mixed the two lines into one group for the interaction tests. The correlation coefficients between the different body weights of different weeks were calculated in the combined population (Table 3). The correlation coefficient between BW0-BW7 was near zero (0.015), indicating that BW0 and BW7 are uncorrelated, which was the minimum in Table 3 . The correlation coefficients between BW0-BW1, BW1-BW3, BW3-BW5, and BW5-BW7 steadily increased and exhibited high values between $0.65-0.69$.

\section{Epistatic analysis of body weight}

MatrixEpistasis [13] is an ultrafast method that performs an exhaustive epistatic scanning for quantitative traits with covariate adjustment, and was applied to the interaction tests. With the significance threshold of $8.67 \times 10^{-12}$ (Bonferroni-adjusted: 1\%), 67 (Table 4), four (Table 5), and two (Table 6) statistically significant SNP pairs associated with $\mathrm{BW} 0, \mathrm{BW} 1$, and $\mathrm{BW} 3$ were detected, respectively, with no replicated significant pairs. There were no SNP pairs with a $p$-value smaller than the threshold in BW5 and BW7.

\section{SNPs' interaction networks}

The SNP-SNP interaction network of BWO is constituted of separated subnets, and the subnets containing more than three nodes are shown in Fig. 1. The SNP epistatic interaction network is approximatively 'small world' and scale-free, both major topological features of interaction networks in biology. 'Small world' means shorter paths and independent subnets, resulting in dense local neighborhoods of genes that interact with each other [1]. The results of gene-gene interaction will be inferred in the next step. The scale-free property of networks implies that Gga_rs14184594 is the hub locus with the maximum degree.

Table 1 Summary of genome-wide markers

\begin{tabular}{|c|c|c|c|c|c|c|c|}
\hline $\mathrm{GGA}^{1}$ & SNPs' number & GGA length (Mb) & Mean distance (kb) & GGA & SNPs' number & GGA length (Mb) & Mean distance $(\mathrm{kb})$ \\
\hline 1 & 7538 & 200.95 & 26.66 & 17 & 922 & 10.61 & 11.51 \\
\hline 2 & 5652 & 154.79 & 27.39 & 18 & 917 & 10.89 & 11.87 \\
\hline 3 & 4322 & 113.65 & 26.30 & 19 & 880 & 9.90 & 11.25 \\
\hline 4 & 3518 & 94.16 & 26.77 & 20 & 1574 & 13.92 & 8.84 \\
\hline 5 & 2295 & 62.23 & 27.11 & 21 & 796 & 6.95 & 8.73 \\
\hline 6 & 1814 & 35.84 & 19.76 & 22 & 327 & 3.89 & 11.90 \\
\hline 7 & 1907 & 38.17 & 20.01 & 23 & 643 & 6.02 & 9.37 \\
\hline 8 & 1486 & 30.62 & 20.61 & 24 & 758 & 6.37 & 8.40 \\
\hline 9 & 1240 & 24.02 & 19.37 & 25 & 181 & 2.02 & 11.17 \\
\hline 10 & 1379 & 22.42 & 16.26 & 26 & 670 & 5.03 & 7.51 \\
\hline 11 & 1312 & 21.87 & 16.67 & 27 & 506 & 4.84 & 9.56 \\
\hline 12 & 1425 & 20.46 & 14.36 & 28 & 607 & 4.47 & 7.37 \\
\hline 13 & 1204 & 18.32 & 15.21 & LGE22 & 115 & 0.88 & 7.67 \\
\hline 14 & 1062 & 15.76 & 14.84 & LEG64 & 3 & 0.02 & 6.80 \\
\hline 15 & 1082 & 12.93 & 11.95 & Z & 2001 & 74.59 & 37.28 \\
\hline 16 & 16 & 0.42 & 26.12 & $U N^{a}$ & 672 & / & / \\
\hline
\end{tabular}

1GGA is an abbreviation for Gallus gallus

${ }^{a}$ These SNPs were not assigned to any chromosomes 
Table 2 The Mean \pm Standard deviation (SD) of the body weight in lean and fat lines, respectively, and in the combined population

\begin{tabular}{llll}
\hline Traits & Combined population (475 birds) & Lean line (203 birds) & Fat line (272 birds) \\
\hline BW0 (g) & $44.76 \pm 3.39$ & $44.83 \pm 2.79$ & $44.70 \pm 3.78$ \\
BW1 (g) & $121.97 \pm 12.34$ & $121.05 \pm 12.80$ & $122.68 \pm 11.95$ \\
BW3 (g) & $615.22 \pm 65.97$ & $617.35 \pm 71.98$ & $613.65 \pm 61.23$ \\
BW5 (g) & $1491.19 \pm 142.53$ & $1487.53 \pm 159.13$ & $1493.91 \pm 129.10$ \\
BW7 (g) & $2400.97 \pm 221.65$ & $2419.53 \pm 246.45$ & $2387.11 \pm 200.51$ \\
\hline
\end{tabular}

\section{Annotation of SNP loci and SNP-SNP interaction networks} In total, 401 genes and 41 microRNAs were annotated to the BW0 network. Furthermore, 25 genes and one microRNA were annotated to the BW1 network and 19 genes and one microRNA to the BW3 network (Additional file 1: Table S1, Additional file 2: Table S2 and Additional file 3: Table S3).

The interaction network feature analysis was applied to the BW0 network, because the interaction network size and topology structure of BW1 and BW3 were small and apparent. The significant interaction SNP-SNP pairs contain 80 single SNPs in which 30 SNPs are located in the $\mathrm{Z}$ chromosome. Observing the annotation information indicated something interesting. Many SNPs from the same subnet are neighbors, concentrating in the same region. Therefore, we adjusted the spatial position of SNPs in Fig. 1, placing SNPs closer together if they were in the same region. All the subnets include SNPs from the same region, except SubNet_8 and SubNet_9. The phenomenon enhanced the reliability to infer that SNP-SNP interactions would be the result of gene-gene interactions in the correspond regions. For example, the cross lines in Subnet_1 accounted for the interactions between chr19: $(3,823,581,5,935,922)$ and chrz: $(65,912$, 281, 67,063,604) and chr19: $(1,728,331,3,504,813)$ and chrz: $(65,912,281,67,063,604)$, which would be the signal of the gene set (INIP, GNG10, SMC2, PTGR1, TXN, $M U S K, L P A R 1)$ on the $\mathrm{Z}$ chromosome interacting with the gene set on the chromosome 19 (CUX1, PRKRIP1, ORAI3 et al.). Furthermore, SubNet_2 claimed the gene set (GLDC, TYRP1, MPDZ, NFIB, ZDHHC21, CER1, $P S I P 1)$ on the $\mathrm{Z}$ chromosome interacting with the gene set (IGFBP1, IGFBP3, TNS3, SLC12A7) near the hub SNP on the chromosome 2. All the inferred gene set interactions are shown in Table 7. However, the

Table 3 The correlation coefficient between body weights in the combined population

\begin{tabular}{llllll}
\hline & BW 0 & BW 1 & BW 3 & BW 5 & BW 7 \\
\hline BW 0 & 1 & 0.357 & 1 & 0.037 & 0.015 \\
BW 1 & & 1 & 0.590 & 0.363 & 0.283 \\
BW 3 & & 1 & 0.694 & 0.436 \\
BW 5 & & & 1 & 0.646 \\
BW 7 & & & & 1 \\
\hline
\end{tabular}

annotation would generate a gene set in each region, thus we could conclude the interaction existing between gene sets, whereas the point-to-point interactive relationship could not be provided.

The interaction effects could be detected in the same or different chromosomes. SubNet_3 and SubNet_4 indicated that the interaction effect could happen within the same chromosome. Furthermore, SNPs in SubNet_4 were all in the same region, neighborhood genes interacting with each other. Other SubNets all illustrated that the interaction effects were present in different chromosomes.

Eight gene ontology terms were significantly enriched, including chromosome, nucleus, phosphoprotein, acetylation, DNA-binding, nucleosome, nucleosome core, and histone H5 (Additional file 4: Table S4). Five pathways were significantly enriched, including the calcium signaling pathway, focal adhesion, ECM-receptor interaction, melanogenesis, and oocyte meiosis (Additional file 5: Table S5).

\section{Discussion}

To our knowledge, this study used a novel approach by detecting the interaction effects that affect the quantitative traits of phenotype variation at multiple time points with SNP data. Based on the recognition that phenotypic data continuously changes, which is a key feature of quantitative traits, we assessed the similarity of the genetic network of quantitative traits at different periods. Many studies have evaluated interaction effects; however, no studies have assessed whether the genetic networks are time-dependent. Our study has demonstrated that genetic networks are time-dependent, contributing to our understanding of this field.

Chicken (Gallus gallus) is a vertebrate, a model organism, and agricultural species, and its body weight is a typical quantitative trait that can be easily measured. Broiler body weight's heritability in males ranges from 0.29 to $0.37[14,15]$, a medium to high level. In the experimental population of this study, body weight's heritability in males ranged from 0.28 to 0.53 . Thus, broiler body weight is a suitable quantitative trait for detecting interaction effects and determining the features of genetic networks.

The male body weight data used in the study were derived from NEAUHLF, a broiler line. Although the 
Table 4 Genome-wide significant pairwise epistatic interactive SNP pairs for BWO

\begin{tabular}{|c|c|c|c|c|}
\hline $\mathrm{GGAF}^{\mathrm{a}}$ & SNP1 name & $\mathrm{GGAS}^{\mathrm{a}}$ & SNP2 name & $P$-val \\
\hline chr1 & Gga_rs10727935 & chr13 & GgaluGA096558 & 82 \\
\hline chr1 & GgaluGA010224 & chr4 & Gga_rs14493884 & \\
\hline chr1 & GgaluGA016211 & chr6 & Gga_rs14588369 & 51 \\
\hline chr1 & GgaluGA016211 & chr7 & Gga_rs14614638 & \\
\hline chr1 & Gga_rs13982417 & chrz & Gga_rs15249625 & 9.59 \\
\hline chr11 & GGaluGA078115 & chrz & Gga_rs14759127 & \\
\hline chr12 & Gga_rs14031249 & chr19 & GgaluGA124944 & \\
\hline chr12 & GgaluGA081258 & chr19 & GgaluGA125308 & \\
\hline chr14 & Gga_rs15719971 & chr20 & Gga_rs14277625 & \\
\hline chr19 & Gga_rs10730456 & chrz & Gga_rs16774940 & \\
\hline chr19 & Gga_rs10730456 & chrz & Gga_rs16774954 & 3 \\
\hline chr19 & Gga_rs10730456 & chrz & Gga_rs14775753 & \\
\hline chr19 & Gga_rs14118327 & chrz & Gga_rs16774940 & \\
\hline chr19 & Gga_rs14118327 & chrz & Gga_rs16774954 & \\
\hline chr19 & Gga_rs14118327 & chrz & Gga_rs14775753 & \\
\hline chr19 & Gga_rs15045504 & chrz & Gga_rs16774954 & \\
\hline chr19 & Gga_rs15045504 & chrz & Gga_rs16774940 & \\
\hline chr19 & Gga_rs15045732 & chrz & Gga_rs16774940 & \\
\hline chr19 & Gga_rs15045732 & chrz & Gga_rs16774954 & \\
\hline chr19 & Gga_rs15045732 & chrz & Gga_rs14775753 & \\
\hline chr19 & Gga_rs15048206 & chrz & Gga_rs16774954 & \\
\hline chr19 & Gga_rs15048206 & chrz & Gga_rs16774940 & 2. \\
\hline chr19 & Gga_rs15048206 & chrz & Gga_rs14775753 & \\
\hline chr19 & Gga_rs15048223 & chrz & Gga_rs16774940 & 5.9 \\
\hline chr19 & Gga_rs15048223 & chrz & Gga_rs16774954 & J. \\
\hline chr19 & GgaluGA126270 & chrz & Gga_rs16774954 & \\
\hline chr19 & GgaluGA126270 & chrz & Gga_rs16774940 & \\
\hline chr2 & GgaluGA160608 & chr6 & GgaluGA295929 & \\
\hline chr2 & Gga_rs14135538 & chrz & Gga_rs14755141 & \\
\hline chr2 & Gga_rs14135538 & chrz & Gga_rs16101791 & 4.79 \\
\hline chr2 & Gga_rs14184594 & chrz & Gga_rs16106712 & \\
\hline chr2 & Gga_rs14184594 & chrz & Gga_rs16065879 & \\
\hline chr2 & Gga_rs14184594 & chrz & Gga_rs16091913 & \\
\hline chr2 & Gga_rs14184594 & chrz & Gga_rs16106257 & \\
\hline chr2 & Gga_rs14184594 & chrz & Gga_rs14738375 & 7.44 \\
\hline chr2 & Gga_rs14184594 & chrz & Gga_rs16071074 & 7 \\
\hline chr2 & Gga_rs14184594 & chrz & Gga_rs16091907 & \\
\hline chr2 & Gga_rs14184594 & chrz & Gga_rs16106786 & 7.4 \\
\hline chr2 & Gga_rs14184594 & chrz & Gga_rs16776264 & \\
\hline chr24 & GgaluGA193154 & chrz & Gga_rs14753903 & 2.79 \\
\hline chr27 & Gga_rs16208036 & chrz & Gga_rs14762941 & \\
\hline chr27 & Gga_rs16208036 & chrz & Gga_rs16108466 & 0.6 \\
\hline chr27 & GgaluGA199670 & chrz & GgaluGA349792 & 7.8 \\
\hline
\end{tabular}

Table 4 Genome-wide significant pairwise epistatic interactive SNP pairs for BWO (Continued)

\begin{tabular}{|c|c|c|c|c|}
\hline $\mathrm{GGAF}^{\mathrm{a}}$ & SNP1 name & $\mathrm{GGAS}^{\mathrm{a}}$ & SNP2 name & $P$-value \\
\hline chr3 & Gga_rs14082553 & chr19 & GgaluGA126270 & $9.81 \mathrm{E}-14$ \\
\hline chr3 & GgaluGA210739 & chr19 & Gga_rs14119969 & $5.99 \mathrm{E}-12$ \\
\hline chr3 & Gga_rs14323198 & chr3 & Gga_rs14394679 & 4.13E-12 \\
\hline chr3 & Gga_rs14323198 & chr3 & Gga_rs14395789 & 4.25E-12 \\
\hline chr3 & Gga_rs15426103 & chr3 & Gga_rs14323198 & 7.57E-12 \\
\hline chr3 & Gga_rs16320563 & chr3 & Gga_rs14323198 & 1.25E-12 \\
\hline chr3 & Gga_rs14385387 & chrz & GgaluGA349792 & 1.91E-12 \\
\hline chr4 & GgaluGA267201 & chr13 & GgaluGA093775 & 3.69E-12 \\
\hline chr4 & GgaluGA268612 & chr4 & Gga_rs16424343 & 4.73E-13 \\
\hline chr4 & 90998 & chr5 & $\mathrm{Gg}$ & 4.32E-12 \\
\hline chr4 & Gga_rs13665914 & chr9 & Gga_rs13608349 & 1.59E-12 \\
\hline chr4 & Gga_rs15474576 & chrz & Gga_rs16108466 & 2.69E-13 \\
\hline chr5 & Gga_rs15736571 & chrz & Gga_rs14767978 & 7.14E-12 \\
\hline chr7 & Gga_rs10728585 & chrz & Gga_r & 2.29E-12 \\
\hline chr7 & Gga_rs15871969 & chrz & Gga_rs14759127 & $1.08 \mathrm{E}-13$ \\
\hline chr7 & Gga_rs15871969 & chrz & Gga_rs14759170 & 2.49E-12 \\
\hline chrz & Gga_rs13816749 & chrz & GgaluGA350520 & 2.63E-12 \\
\hline chrz & Gga_rs14691748 & chrz & GgaluGA350520 & 7.93E-13 \\
\hline chrz & Gga_rs14762941 & chrz & Gga_rs16763798 & 8.37E-12 \\
\hline chrz & Gga_rs16080645 & chrz & GgaluGA350520 & 2.63E-12 \\
\hline chrz & Gga_rs16685135 & chrz & GgaluGA350520 & 2.63E-12 \\
\hline chrz & Gga_rs16763798 & chrz & Gga_rs16108466 & $1.92 \mathrm{E}-12$ \\
\hline chrz & Gga_rs16764173 & chrz & Gga_rs16763798 & 7.77E-12 \\
\hline chrz & Gga_rs16764637 & chrz & Gga_rs16764173 & 1.60E-12 \\
\hline
\end{tabular}

${ }^{\mathrm{a}} \mathrm{GGAF}=$ The first chromosome in the pairwise epistasis analysis; GGAS = The second chromosome in the pairwise epistasis analysis; $P$-value $=P$-value of the effect being tested

resource population contains both the lean and fat lines, we decided to use all the samples in the current study. The decision was based on the selection of population, birds breeding, and phenotypic value's statistical character. To be specific, the two lines had to come from the same grandsire line and be raised under the same environmental conditions. Body weights of male birds in the second hatch were considered. As a result, body weights did not exhibit significant differences between the lean

Table 5 Genome-wide significant pairwise epistatic interactive SNP pairs for BW1

\begin{tabular}{lllll}
\hline GGAF $^{\text {a }}$ & SNP1 name & GGAS $^{\text {a }}$ & SNP2 name & $P$-value \\
\hline chr1 & GgaluGA061615 & chr4 & Gga_rs14491923 & $2.39 \mathrm{E}-12$ \\
chr2 & GgaluGA136764 & chr3 & GgaluGA211595 & $3.79 \mathrm{E}-13$ \\
chr2 & GgaluGA133839 & chr5 & Gga_rs13756660 & $2.41 \mathrm{E}-12$ \\
chr2 & GgaluGA133839 & chr5 & Gga_rs14515483 & $8.44 \mathrm{E}-12$ \\
\hline
\end{tabular}

${ }^{a} \mathrm{GGAF}=$ The first chromosome in the pairwise epistasis analysis; GGAS = The second chromosome in the pairwise epistasis analysis; $P$-value $=P$-value of the effect being tested 
Table 6 Genome-wide significant pairwise epistatic interactive SNP pairs for BW3

\begin{tabular}{lllll}
\hline GGAF $^{\text {a }}$ & SNP1 name & GGAS $^{\text {a }}$ & SNP2 name & P-value \\
\hline chr5 & GgaluGA273676 & chr11 & Gga_rs14965049 & $2.80 \mathrm{E}-13$ \\
chr7 & Gga_rs13598324 & chr12 & Gga_rs14045047 & 3.88E-12
\end{tabular}

${ }^{\mathrm{a}} \mathrm{GGAF}=$ The first chromosome in the pairwise epistasis analysis; GGAS = The second chromosome in the pairwise epistasis analysis; $P$-value $=P$-value of the effect being tested

and fat lines (Table 2). More importantly, larger sample numbers improve the interaction test power. We performed the test in the lean and fat lines separately, yet no significant SNP pairs were detected. Thus, the phenotypic values were not divided according to the line.

In the previous study, pair-wise interaction effects associated with important traits in chickens have been identified by the EPISNP3 module in epiSNP_v4.2_Windows software package $[12,16]$. However, no significant epistatic interactions affecting body weight (BW1, BW3, BW5, and BW7) were detected. In the current study, we focused on bird's body weight. From day of hatch to 7 weeks, the phenotypic data contained five time points, which were treated as time series data. Furthermore, the new method MatrixEpistasis, which can remove confounding bias through covariate adjustment, was used [13]. Population genomic stratification might occur in the long-term artificially selected population due to selection pressure. MatrixEpistasis can handle this bias and offers another advantage of ultra-computational speed, the critical factor for SNP-SNP interaction mapping at the genome-wide level. With the new method, we found some interesting results.

Testing multiple hypotheses caused that the significance threshold $p$-value $\left(8.67 \times 10^{-12}\right.$, Bonferroniadjusted: $1 \%$ ) was far smaller than $1 \%$. The significant test results heavily depended on the arbitrary significance threshold. Although some effects were thus ignored, the strict threshold enhanced the confidence of our results. With the strict threshold, the detection results showed that the interaction effect was completely different at different time points. This suggests that the time point is an important factor in the quantitative trait interaction test. It is easy to determine the interaction effect on the day of hatch, whereas it is difficulty at 5 and 7 weeks. This demonstrates that the cooperation between genes is closer in the early weeks than later weeks. From the perspective of data driving, the correlations between the body weight at BW0 and other weeks were relatively small, which can partially explain the different results. Furthermore, the results indicate that the genetic regulation networks are different at different time

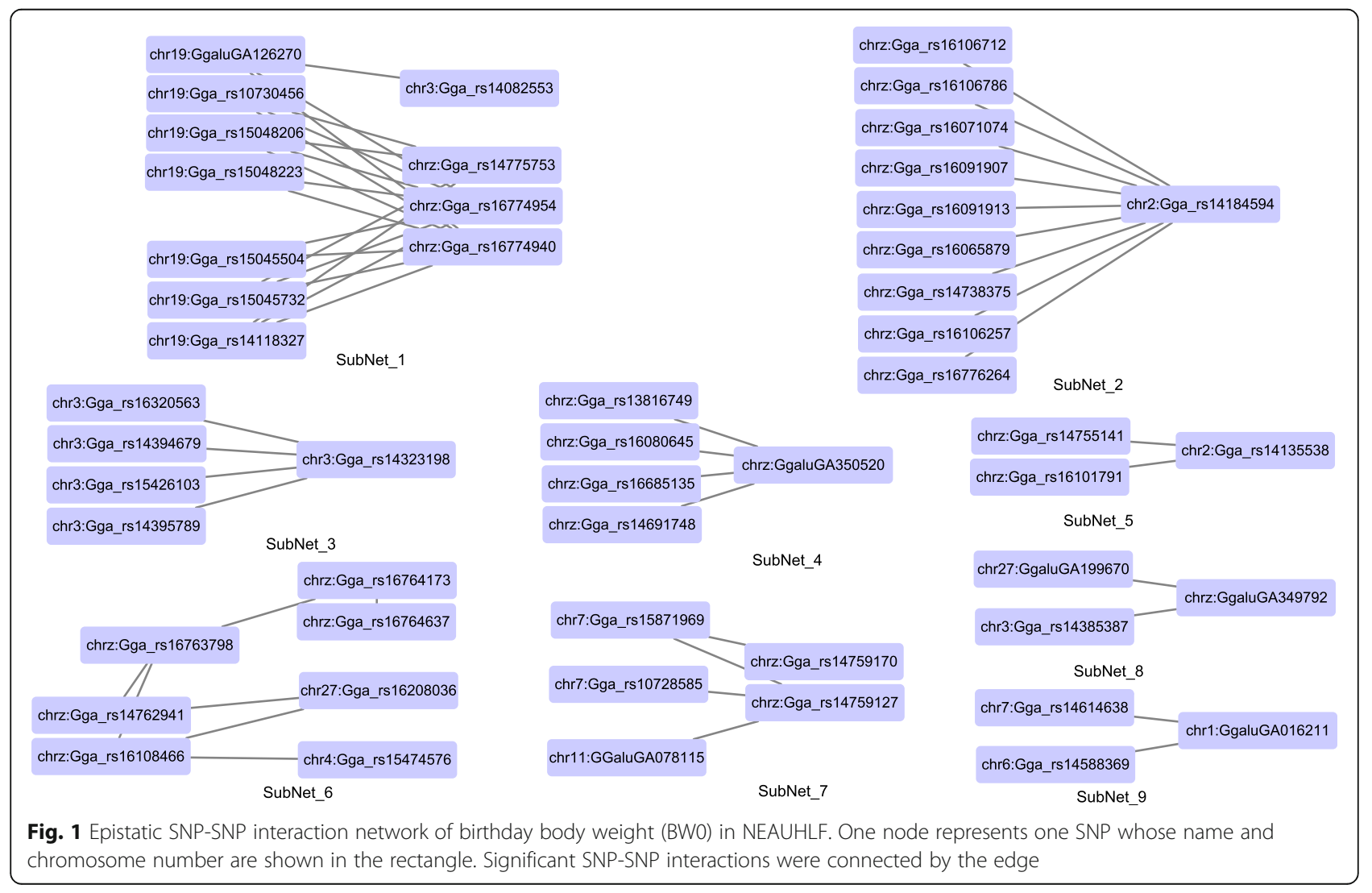


Table 7 Gene sets interaction inferred from the network of BW0

\begin{tabular}{|c|c|c|}
\hline SubNet & Gene set $\mathrm{A}$ & Gene set B \\
\hline 1 & $\begin{array}{l}\text { CUX1, PRKRIP1, ORAI3, RASA4, YWHAG, HSPB1, POR, RAD51D, RFFLL LIG3, } \\
\text { CCLI5, CCL1, CCAH221, MRPS17, NIPSNAP2, PSPH, CCT6A, PHKG1, CHCHD2, } \\
\text { VKORC1L1, GUSB, ASL2, ASL1, CRCP, KCTD7, NCBP3, CRK, YWHAE, MYO1C, } \\
\text { INPPSK, SERPINF1, SMYD4, RPA1, DPH1, HIC1, POLDIP2, VTN, SLC46A1, } \\
\text { ALDOC, TLCD1, FAM222B, ERAL1, FLOT2 }\end{array}$ & INIP, GNG10, SMC2, PTGR1, TXN, MUSK, LPAR1 \\
\hline 1 & $\begin{array}{l}\text { CUX1, PRKRIP1, ORAI3, RASA4, YWHAG, HSPB1, POR, RAD51D, RFFL, LIG3, } \\
\text { CCLI5, CCL1, CCAH221, MRPS17, NIPSNAP2, PSPH, CCT6A, PHKG1, CHCHD2, } \\
\text { VKORCIL1, GUSB, ASL2, ASL1, CRCP, KCTD7, NCBP3, CRK, YWHAE, MYO1C, } \\
\text { INPP5K, SERPINF1, SMYD4, RPA1, DPH1, HIC1, POLDIP2, VTN, SLC46A1, } \\
\text { ALDOC, TLCD1, FAM222B, ERAL1, FLOT2 }\end{array}$ & $\begin{array}{l}\text { DTD1, SEC23B, DZANK1, BIRC5, POLR3F, KAT14, MGME1, SNX5, RRBP1, } \\
\text { DSTN, CST3, CST7, APMAP, TTBK1, SLC22A7, TTL, VSX1, ENTPD6, MAL, } \\
\text { MRPS5, SLC8A1 }\end{array}$ \\
\hline 1 & $\begin{array}{l}\text { CASTOR2, RCC1L, NCF1, RFC2, LAT2, EIF4H, UBE2G1, ATP2A3, P2RX1, MIS12, } \\
\text { RABEP1 }\end{array}$ & INIP, GNG10, SMC2, PTGR1, TXN, MUSK, LPAR1 \\
\hline 2 & GLDC, TYRP1, MPDZ, NFIB, ZDHHC21, CER1, PSIP1 & IGFBP1, IGFBP3, TNS3, SLC12A7 \\
\hline 3 & MYOM2, CLN8, ERICH1, ACP1, TMEM18 & GPATCH2, ESRRG \\
\hline 4 & GLDC, TYRP1, MPDZ, NFIB, ZDHHC21, CER1, PSIP1 & GLDC, TYRP1, MPDZ, NFIB, ZDHHC21, CER1, PSIP1 \\
\hline 5 & INSIG1, EN2, SHH, LMBR1, MNX1, UBE3C, DNAJB6 & SLC30A5, CENPH, THBS4, MTX3 \\
\hline 6 & S100Z, F2RL1, IQGAP2, POLK, HMGCR, NSA2, UTP15, ANKRA2, FOXD1 & ITGAI, PELO, FST \\
\hline 6 & ITGA1, PELO, FST & $N F I B, Z D H H C 21, C E R 1, P S I P 1$ \\
\hline 6 & $\begin{array}{l}\text { MRPL45, CBX1, NFE2L1, CDK5RAP3, LOC107055293, PCGF2, RPL23, LASP1, } \\
\text { RPL19, ERBB2, IKZF3, ZPBP2, GSDMA, PSMD3, CSF3, MED24, THRA, RARA, } \\
\text { TOP2A, IGFBP4, CCR7, SMARCE1, KRT222, KRT20 }\end{array}$ & $N F I B, Z D H H C 21, C E R 1, P S I P 1$ \\
\hline 6 & $\begin{array}{l}\text { BTK, TIMM8A, TAF7L, CENPI, DKC1, MPP1, RHOGL, NONO, GJB1, NLGN3, } \\
\text { IL2RG, SNX12, LOC422214, HTR2C, IL13RA2, PLS3 }\end{array}$ & $N F I B, Z D H H C 21, C E R 1, P S I P 1$ \\
\hline 7 & S100Z, F2RL1, IQGAP2, POLK, HMGCR, NSA2 & SEC22A, ADCY5, HACD2, MYLK \\
\hline 7 & METTL21A, CREB1, KLF7, ADAM23, EEF1B2, NDUFS1, NRP2 & S100Z, F2RL1, IQGAP2, POLK, HMGCR, NSA2 \\
\hline 7 & S100Z, F2RL1, IQGAP2, POLK, HMGCR, NSA2 & NUDT7 \\
\hline 8 & $\begin{array}{l}\text { ACE, KCNH6, DCAF7, LIMD2, RNF113A, STRADA, DDX42, MYL4, CDC27, } \\
\text { MAPT, ITGA3, DLX3, KAT7, SLC35B1, NGFR, MEOX1, PHB }\end{array}$ & POLK, HMGCR, NSA2, UTP15, ANKRA2, FOXD1 \\
\hline 8 & TMEM30A, COX7A2, COL12A1, SLC17A5, EEF1A1, MTO1 & POLK, HMGCR, NSA2, UTP15, ANKRA2, FOXD1 \\
\hline 9 & $\begin{array}{l}\text { ARL1, CNOT4, WDR91, PDE6H, ARHGDIB, MGP, OC3, ART4, HIST1H2B7, } \\
\text { HIST1H46L2, HISTH2A4L1, HIST2H4B, HIST1H46, HIST1H2B7, HIST1H110, } \\
\text { HIST1H46L2, HIST1H2B8, HIST2H3L, H2AFJ, HIST1H103, HIST1H2A4, } \\
\text { HIST1H101, HIST1H111L, HIST1H2A4L3, HIST1H2B5, HIST1H3H, HIST1H111R, } \\
\text { DDX47, HEBP1, FAM234B, EMP1 }\end{array}$ & $I F I H 1, F A P, G C G, D P P 4$ \\
\hline 9 & $\begin{array}{l}\text { ARL1, CNOT4, WDR91, PDE6H, ARHGDIB, MGP, OC3, ART4, HIST1H2B7, } \\
\text { HIST1H46L2, HISTH2A4L1, HIST2H4B, HIST1H46, HIST1H2B7, HIST1H110, } \\
\text { HIST1H46L2, HIST1H2B8, HIST2H3L, H2AFJ, HIST1H103, HIST1H2A4, } \\
\text { HIST1H101, HIST1H111L, HIST1H2A4L3, HIST1H2B5, HIST1H3H, HIST1H111R, } \\
\text { DDX47, HEBP1, FAM234B, EMP1 }\end{array}$ & TECTB, ACSL5, VTI1A, TCF7L2, HABP2, DCLRE1A, NHLRC2 \\
\hline
\end{tabular}

points. Carlborg et al. [17] have found a similar conclusion in chicken.

Many SNP positions on the chromosome are neighboring in the SNP-SNP interaction network. We suggest it should be the signal of gene-gene interactions in the corresponding regions.

In this study, we detected 55 regions on 17 chromosomes. Based on the literature, some genes are associated with body weight; for example, IGFBP1 is associated with body weight in the Jinghai Yellow chicken [18]. MuSK was abundantly expressed in the muscle of early-stage fowl embryos [19]. ADCY5 is related to obesity in men and mice [20]. PHKG1 is important in pig skeletal muscle [21]. We identified five pathways related to body weight. The focal adhesion signal pathway plays an important role in the development of chicken muscle [22]. The focal adhesion and ECM-receptor interaction signal pathways are related to intramuscular fat content [23]. In addition, numerous genes are associated with human complex diseases, such as ATP2A3 [24], ITGA1 [25], and THBS4 [26].

Linkage disequilibrium and quantitative trait locus information were not introduced in this study, because linkage disequilibrium testing is not correlated with epistatic interaction tests and gene-gene results are usually more accurate than quantitative trait locus interactions. In fact, gene-gene interaction results were based on the 
gene sets, and the actual gene-gene interactions could be verified by biological experiments.

In the future, more research will be needed to better understand the genetic network of quantitative traits.

\section{Conclusions}

The interaction effect is most active on the day of hatch, after which the effects decline, and by 5-7 weeks the effects are hardly detected. No significant SNP pairs recurred at different time points. Our study demonstrated that the genetic interaction network of chicken body weight is time-dependent and the epistatic interaction effect is dynamic. For the first three time points, the interaction networks indicated that SNP-SNP interactions were concentrated in some special regions on the chromosomes, which were the results of gene-gene interactions. To our knowledge, we are the first to describe and summarize the significant interaction effects that affect chicken body weight variation.

\section{Methods}

\section{Experimental population}

A total of 475 male chickens derived from the 11th generation population of the Northeast Agricultural University broiler lines divergently selected for abdominal fat content (NEAUHLF) were used in the study.

The G0 generation was selected in 1996 and came from the same grandsire line, which originated from the Arbor Acres broiler. According to their plasma very low-density lipoprotein (VLDL) concentration at 7 weeks of age, the G0 birds were divided into two lines, the lean line and the fat line. For each line, 25 half-sib families, with an average of 70 G1 offspring per family in two hatches, were produced by mating the G0 birds (one sire: four dams). From G1 to G11, the birds were raised in two hatches. Abdominal fat percentage (AFP $=a b-$ dominal fat weight/body weight, measured at 7 weeks of age) of the male birds in the first hatch was used as the artificial selection criterion for NEAUHLF. The families' sib birds, with lower (lean line) or higher (fat line) AFP than the population's average value, were selected as candidates for breeding, taking into consideration the plasma VLDL concentration, the body weights of male birds in the second hatch, and the egg production of female birds in both hatches. G11 contained 203 chickens in the lean line and 272 chickens in the fat line.

All the birds were fed with a corn-soybean-based diet that met all National Research Council (NRC) requirements and were raised under the same environmental conditions with free access to feed and water. From hatch to 3 weeks of age, the birds received a starter feed $(3000 \mathrm{kal} \mathrm{ME}=\mathrm{kg}$ and $210 \mathrm{~g}=\mathrm{kg} \mathrm{CP})$. From 4 weeks of age to slaughter, the birds were fed a grower diet $(3100 \mathrm{kal} \mathrm{ME}=\mathrm{kg}$ and $190 \mathrm{~g}=\mathrm{kg} \mathrm{CP})$.
The birds were weighed at day of hatch and at 1, 3, 5 , and 7 weeks of age $[11,12]$.

\section{SNP genotyping and phenotypic values}

Genotyping was carried out using chicken $60 \mathrm{~K}$ SNP chip (57,636 SNPs) manufactured by the Illumina Inc. (San Diego, CA). Monomorphic or minor allele frequencies $(<5 \%)$ loci were filtered out. Individuals whose missing SNP genotypes were $\geq 5 \%$ were removed. After quality control, SNPs with chromosome position information were selected for the interaction analysis. Phenotypic values were analyzed with descriptive statistics. The correlation coefficients of body weight among different weeks age were calculated in the combined population.

\section{Genome-wide pairwise interaction analysis}

The method for detecting the interaction effect was implemented in $\mathrm{R}$ and is available at https://github.com/ fanglab/MatrixEpistasis. The statistical model was:

$$
p=\alpha+\beta_{1} G_{. s}+\beta_{2} G_{. t}+\beta_{3} G_{. s} G_{. t}+\sum_{v} \gamma_{v} C_{. v}+\varepsilon
$$

Where $\alpha$ is the overall mean of the quantitative phenotype; $\beta_{1} / \beta_{2}, \beta_{3}$ and $\gamma_{v}$ are the regression coefficients for the main genetic additive effect, interaction effect, and covariates, respectively, and $\varepsilon$ is a normal variable with zero mean and $\xi^{2}$ variance [13]. In this model, the phenotype has both main genetic additive effects and covariates adjusted, and the size of the interaction effect is the regression coefficient $\beta_{3}$. Therefore, the hypotheses are $\mathrm{H}_{0}: \beta_{3}=0$ and $\mathrm{H}_{1}: \beta_{3} \neq 0$; the tests of interaction correspond to testing whether the regression coefficient $\beta_{3}$ equals zero or not.

MatrixEpistasis [13] was used to identify pairwise (two-dimension, SNP-SNP) significant epistatic interaction effects affecting body weight variation. The significance threshold was set at $8.67 \times 10^{-12}$ (Bonferroniadjusted: $1 \%)$.

\section{SNP-SNP interaction network}

The plots illustrating the SNP-SNP interaction networks with the significant epistatic effects for chicken body weight were drawn with the Cytoscape 3.7.0 software package [14]. The detailed network analysis was mainly applied to the BW0 network, and the BW1 and BW3 networks were sketched, because they were obvious and apparent.

\section{Annotation of the SNP-SNP interaction networks}

For annotating genes to the interaction networks, a 1 $\mathrm{Mb}$ length region was designated for each SNP, $0.5 \mathrm{Mb}$ upstream and $0.5 \mathrm{Mb}$ downstream. The regions were merged if the distance between SNPs was $<1 \mathrm{Mb}$. Genes 
overlapping the regions were retrieved from UCSC (https://genome.ucsc.edu/) (Galgal5). Genes in the same region were put together (the so-called "gene set"). Gene interactions in gene sets were deduced if the significant SNP pairs were located in the corresponding regions.

Functional annotation of genes was performed by DAVID bioinformatics resources 6.8 (https://david.ncifcrf. gov/home.jsp) for gene ontology terms. KOBAS2.0 [27] was used for Kyoto Encyclopedia of Genes and Genomes (KEGG) pathway analysis. The significance threshold was set to the corrected $p$-value $<0.05$.

\section{Supplementary information}

Supplementary information accompanies this paper at https://doi.org/10. 1186/s12864-019-6132-0.

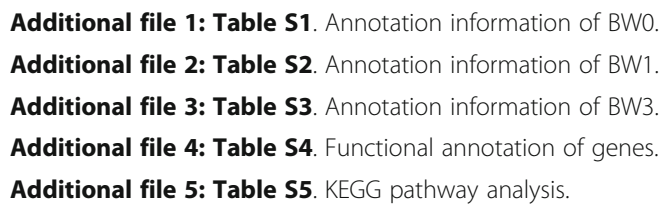

\section{Abbreviations}

GO: Gene ontology; GWAS: Genome-wide association study; KEGG: Kyoto encyclopedia of genes and genomes; LD: Linkage disequilibrium; QTL: Quantitative trait loci; SNP: Single nucleotide polymorphism

\section{Acknowledgements}

The authors would like to thank the members of the Poultry Breeding Group of the College of Animal Science and Technology at the Northeast Agricultural University for managing the birds and collecting the data.

\section{Authors' contributions}

FGL analyzed and interpreted the data as well as drafted and wrote the manuscript. HL led the conception and design of the study and helped write the manuscript. All authors submitted comments on drafts and read and approved the final manuscript.

\section{Funding}

This research was supported by the National Natural Science Foundation (No. 31601935) and China Agriculture Research System (No. CARS-41). The funding bodies did not influence the design of the study, data collection, analysis, or interpretation, or writing of the manuscript.

\section{Availability of data and materials}

The chicken $60 \mathrm{k}$ SNP data used in this article, including the additional files, have been deposited into Gene Expression Omnibus (http://www.ncbi.nlm. nih.gov/geo/) with the identifier GSE58551.

\section{Ethics approval and consent to participate}

All the animal work, the care and use of experimental animals, followed the guidelines established by the Ministry of Science and Technology of the People's Republic of China (Approval number: 2006-398) and were approved by the Laboratory Animal Management Committee of the Northeast Agricultural University.

\section{Consent for publication}

Not applicable.

\section{Competing interests}

The authors declare that they have no competing interests.

\section{Author details}

${ }^{1}$ College of Science, Northeast Agricultural University, Harbin 150030, People's Republic of China. ${ }^{2}$ Key Laboratory of Chicken Genetics and
Breeding, Ministry of Agriculture and Rural Affairs, Harbin 150030, People's Republic of China. ${ }^{3}$ Key Laboratory of Animal Genetics, Breeding and Reproduction, Education Department of Heilongjiang Province, Harbin 150030, People's Republic of China. ${ }^{4}$ College of Animal Science and Technology, Northeast Agricultural University, Harbin 150030, People's Republic of China.

Received: 5 May 2019 Accepted: 23 September 2019

Published online: 23 October 2019

\section{References}

1. Mackay TFC. Epistasis and quantitative traits: using model organisms to study gene-gene interactions. Nat Rev Genet. 2014;15(1):22.

2. Kauffman SA. The origins of order: self-organization and selection in evolution. New York: Oxford University Press; 1993.

3. Huang W, Richards S, Carbone MA, et al. Epistasis dominates the genetic architecture of Drosophila quantitative traits. Proc Natl Acad Sci. 2012; 109(39):15553-9.

4. Hoffmann TJ, Theusch E, Haldar T, et al. A large electronic-health-recordbased genome-wide study of serum lipids. Nat Genet. 2018;50:401-413.

5. Manolio TA, Collins FS, Cox NJ, et al. Finding the missing heritability of complex diseases. Nature. 2009:461(7265):747-53.

6. Wood AR, Esko T, Yang J, Vedantam S, Pers TH, Gustafsson S, Chu AY, Estrada K, Luan J, Kutalik Z, et al. Defining the role of common variation in the genomic and biological architecture of adult human height. Nat Genet. 2014;46(11):1173-86.

7. Eichler EE, Flint J, Gibson G, et al. Missing heritability and strategies for finding the underlying causes of complex disease. Nat Rev Genet. 2010; 11(6):446-50.

8. Zuk O, Hechter E, Sunyaev SR, et al. The mystery of missing heritability: genetic interactions create phantom heritability. Proc Natl Acad Sci. 2012; 109(4):1193-8.

9. Andersson L. CarlborgO, Jacobsson L, et al. epistasis and the release of genetic variation during long-term selection. Nat Genet. 2006;38(4): 418-20.

10. Hu G, Wang SZ, Wang ZP, et al. Erratum to "genetic epistasis analysis of 10 peroxisome proliferator-activated receptor $\gamma$-correlated genes in broiler lines divergently selected for abdominal fat content" (Poult. Sci. 89:2341-2350). Poult Sci. 2010:89(11):2341-50.

11. Li F, Hu G, Zhang H, et al. Epistatic effects on abdominal fat content in chickens: results from a genome-wide SNP-SNP interaction analysis. PLoS One. 2013;8(12):e81520.

12. Zhang $\mathrm{H}$, Yu JQ, Yang LL, et al. Identification of genome-wide SNP-SNP interactions associated with important traits in chicken. BMC Genomics. 2017;18(1):892.

13. Zhu S, Fang G, Berger B. MatrixEpistasis: ultrafast, exhaustive epistasis scan for quantitative traits with covariate adjustment. Bioinformatics. 2018;34(14): 2341-2348.

14. Maniatis G, Demiris N, Kranis A, et al. Comparison of inference methods of genetic parameters with an application to body weight in broilers. Archives Animal Breeding. 2015;58(2):277-86.

15. Mebratie W, Madsen P, Hawken R, et al. Multi-trait estimation of genetic parameters for body weight in a commercial broiler chicken population. Livest Sci. 2018;217:15-8.

16. Ma L, Runesha HB, Dvorkin D, et al. Parallel and serial computing tools for testing single-locus and epistatic SNP effects of quantitative traits in genome-wide association studies. Bmc Bioinformatics. 2008;9(1):315.

17. Carlborg O. A global search reveals epistatic interaction between QTL for early growth in the chicken. Genome Res. 2003;13(3):413-21.

18. Zhao X, Wang J, Zhang G, et al. Polymorphisms in exons of the IGFBP-1 gene and their associations with body weight in the Jinghai yellow chicken. Anim Sci Paper Rep. 2013;31(1):55-62.

19. Ip F, Glass D, Gies D, et al. Cloning and characterization of muscle-specific kinase in chicken. Mol Cell Neurosci. 2000;16(5):661-73.

20. Knigge A, Klöting N, Schön MR, et al. ADCY5 Gene Expression in Adipose Tissue Is Related to Obesity in Men and Mice. PLOS ONE. 2015;10(3): e0120742.

21. Ma J, Yang J, Zhou L, et al. A splice mutation in the PHKG1 gene causes high glycogen content and low meat quality in pig skeletal muscle. PLoS Genet. 2014;10(10):e1004710. 
22. Longzhou L, Zhong L, Huayun H, et al. Study on The Related Genes Expression of Focal Adhesion Signal Pathway in Development of Chicken Muscle. China Poultry. 2018;40(17):8-12.

23. Cesar ASM, Regitano LCA, Koltes JE, et al. Putative regulatory factors associated with intramuscular fat content. PLoS One. 2015;10(6):e0128350.

24. Korosec B, Glavac D, Volavsek M, et al. ATP2A3 gene is involved in cancer susceptibility. Cancer Genet Cytogenet. 2009;188(2):88-94.

25. Yim DH, Zhang YW, Eom SY, et al. ITGA1 polymorphisms and haplotypes are associated with gastric cancer risk in a Korean population. World $J$ Gastroenterol. 2013;19(35):5870-6.

26. Chen $X$, Huang $Y$, Wang $Y$, et al. THBS4 predicts poor outcomes and promotes proliferation and metastasis in gastric cancer. J Physiol Biochem. 2019;75(1): $117-123$.

27. Xie C, Mao X, Huang J, et al. KOBAS 2.0: a web server for annotation and identification of enriched pathways and diseases. Nucleic Acids Res. 2011; 39(suppl):W316-22.

\section{Publisher's Note}

Springer Nature remains neutral with regard to jurisdictional claims in published maps and institutional affiliations.

Ready to submit your research? Choose BMC and benefit from:

- fast, convenient online submission

- thorough peer review by experienced researchers in your field

- rapid publication on acceptance

- support for research data, including large and complex data types

- gold Open Access which fosters wider collaboration and increased citations

- maximum visibility for your research: over $100 \mathrm{M}$ website views per year

At BMC, research is always in progress.

Learn more biomedcentral.com/submissions 